\title{
PID Tuning: Robust and Intelligent Multi-Objective Approaches
}

\author{
Hassan Bevrani ${ }^{1}$ and Hossein Bevrani ${ }^{2}$ \\ ${ }^{1}$ University of Kurdistan, \\ ${ }^{2}$ University of Tabriz, \\ Iran
}

\section{Introduction}

The proportional-integral-derivative (PID) control structures have been widely used in industrial applications due to their design/structure simplicity and inexpensive cost. The success of the PID controllers depends on the appropriate choice of their parameters. In practice, tuning the PID parameters/gains is usually realized by classical, trial-and-error approaches, and experienced human experts, which they may not capable to achieve a desirable performance for complex real-world systems with high-order, time-delays, nonlinearities, uncertainties, and without precise mathematical models.

On the other hand, the most of real-world control problems refer to multi-objective control designs that several objectives such as stability, disturbance attenuation and reference tracking with considering practical constraints must be simultaneously followed by a controller. In such cases, using a single norm based performance criteria to evaluate the robustness of resulted PID-based control systems is difficult and multi-objective tuning solutions are needed.

This chapter introduces three effective robust and intelligent multi-objective methodologies for tuning of PID controllers to improve the performance of the closed-loop systems in comparison of conventional PID tuning approaches. The introduced tuning strategies are based on mixed $\mathrm{H}_{2} / \mathrm{H}_{\infty}$, multi-objective genetic algorithm (GA), fuzzy logic, and particle swarm optimization (PSO) techniques. Indeed, these robust and intelligent techniques are employed as optimization engines to produce the PID parameters in the control loops with performance indices near to the optimal ones.

Numerical examples on automatic generation control (AGC) design in multi-area power systems are given to illustrate the mentioned methodologies. It has been found that the controlled systems with proposed PID controllers have better capabilities of handling the large scale and complex dynamical systems.

\section{Mixed $\mathrm{H}_{2} / \mathrm{H}_{\infty}$-based PID tuning}

Mixed $\mathrm{H}_{2} / \mathrm{H}_{\infty}$ provides a powerful control design to meet different specified control objectives. However, it is usually complicated and not easily implemented for the real industrial applications. Recently, some efforts are reported to make a connection between the theoretical mixed $\mathrm{H}_{2} / \mathrm{H}_{\infty}$ optimal control and simple classical PID control (Takahashi et 
al., 1997; Chen et al., 1998; Bevrani \& Hiyama, 2007). (Takahashi et al., 1997) has used a combination of different optimization criteria through a multiobjective technique to tune the PI parameters. A genetic algorithm (GA) approach to mixed $\mathrm{H}_{2} / \mathrm{H}_{\infty}$ optimal PID control is given in (Chen et al., 1998). (Bevrani \& Hiyama, 2007) has addressed a new method to bridge the gap between the power of optimal mixed $\mathrm{H}_{2} / \mathrm{H}_{\infty}$ multiobjective control and PI/PID industrial controls. In this work, the PI/PID control problem is reduced to a static output feedback control synthesis through the mixed $\mathrm{H}_{2} / \mathrm{H}_{\infty}$ control technique, and then the control parameters are easily carried out using an iterative linear matrix inequalities (ILMI) algorithm.

In this section, based on the idea given in (Bevrani \& Hiyama, 2007), the interesting combination of different objectives including $\mathrm{H}_{2}$ and $\mathrm{H}_{\infty}$ tracking performances for a PID controller has been addressed by a systematical, simple and fast algorithm. The multiobjective PID control problem is formulated as a mixed $\mathrm{H}_{2} / \mathrm{H}_{\infty}$ static output feedback (SOF) control problem to obtain a desired PID controller. The developed ILMI algorithm in (Bevrani \& Hiyama, 2007) is used to tune the PID control parameters to achieve mixed $\mathrm{H}_{2} / \mathrm{H}_{\infty}$ optimal performance.

\subsection{PID as a SOF control}

Consider a general system $(\mathrm{G}(\mathrm{s}))$ with $u$ and $y_{o}$ variables as input and output signals. Assume that it is desirable to stabilize the system using a PID controller. Here, it will be shown that the PID control synthesis can be easily transferable to a SOF control problem. The main merit of this transformation is in possibility of using the well-known SOF control techniques to calculate the fixed gains, and once the SOF gain vector is obtained, the PID gains are ready in hand and no additional computation is needed.

In a given PID-based control system, the measured output signal $\left(y_{o}\right)$ performs the input signal for the controller which can be written as follows

$$
u=k_{P} y_{o}+k_{I} \int y_{o} d \tau+k_{D} \frac{d y_{o}}{d t}
$$

where $k_{P}, k_{I}$ and $k_{D}$ are constant real numbers. Therefore, by generalizing the system description to include the $y_{o}$, its integral and derivative as a new measured output vector $(y)$, the PID control problem becomes one of finding a SOF that satisfied the prescribed performance requirements. In order to change (1) to a simple SOF control as

$$
u=K y
$$

Equation (1) can be written as follows

$$
u=\left[\begin{array}{lll}
k_{P} & k_{I} & k_{D}
\end{array}\right]\left[\begin{array}{lll}
y_{o} & \int y_{o} d \tau & \left.\frac{d y_{o}}{d t}\right]^{T}
\end{array}\right.
$$

Therefore, $y$ in (2) can be generalized to the following form (Fig. 1).

$$
y=\left[\begin{array}{lll}
y_{o} & \int y_{o} d \tau & \frac{d y_{o}}{d t}
\end{array}\right]^{T}
$$


Since, the ideal differentiator used in (1), (3) and (4) is unrealizable, a real differentiator should be applied in practice. Although most of PID controllers in use have the derivative part switched off, proper use of the derivative action can improve the stability and help maximize the integral gain for a better performance. For real implementation, ideal differintiator $\left(k_{D} s\right)$ can be approximated as $\left(k_{D S} /\left(\lambda k_{D S}+1\right)\right.$, where $\lambda$ is a small number. The effect of real and approximated differentiator on the closed-loop dynamics are discussed in PID control literature.

\subsection{ILMI-based $\mathrm{H}_{2} / \mathrm{H}_{\infty}$ SOF design}

A general control scheme using mixed $\mathrm{H}_{2} / \mathrm{H}_{\infty}$ control technique is shown in Fig. 2. $\mathrm{G}(\mathrm{s})$ is a linear time invariant system with the given state-space realization in (5). The matrix coeificients are constants and it is assumed the system to be stabilizable via a SOF system. Here, $x$ is the state variable vector, $w$ is disturbance and other external input vector, $y$ is the augmented measured output vector and $K$ is the controller. The output channel $z_{2}$ is associated with the LQG aspects $\left(\mathrm{H}_{2}\right.$ performance) while the output channel $z_{\infty}$ is associated with the $\mathrm{H}_{\infty}$ performance.

$$
\begin{aligned}
& \dot{x}=A x+B_{1} w+B_{2} u \\
& z_{\infty}=C_{\infty} x+D_{\infty 1} w_{i}+D_{\infty 2} u \\
& z_{2}=C_{2} x+D_{21} w+D_{22} u \\
& y=C_{y} x+D_{y 1} w
\end{aligned}
$$

Assume $T_{z_{\infty} w}$ and $T_{z_{2} w}$ are the transfer functions from $w$ to $z_{\infty}$ and $w$ to $z_{2}$, respectively; and consider the following state-space realization for the closed-loop system. After defining the appropriate $\mathrm{H}_{\infty}$ and $\mathrm{H}_{2}$ control outputs $\left(z_{\infty}\right.$ and $\left.z_{2}\right)$ for the system, it will be easy to determine matrix coefficients $\left(C_{\infty}, D_{\infty 1}, D_{\infty 2}\right)$ and $\left(C_{2}, D_{21}, D_{22}\right)$.

$$
\begin{aligned}
& \dot{x}=A_{c} x_{i}+B_{I_{c}} w \\
& z_{\infty}=C_{\infty c} x+D_{\infty_{c}} w \\
& z_{2}=C_{2 c} x+D_{2_{c}} w \\
& y=C_{y_{c}} x+D_{y_{c}} w
\end{aligned}
$$

A mixed $\mathrm{H}_{2} / \mathrm{H}_{\infty}$ SOF control design can be expressed as following optimization problem:

Optimization problem: Determine an admissible SOF law $K$, belong to a family of internally stabilizing SOF gains $K_{\text {sof }}$,

$$
u=K y, K \in K_{\text {sof }}
$$

such that

$$
\inf _{K \in K_{s o f}}\left\|T_{z_{2} w_{2}}\right\|_{2} \text { subject to }\left\|T_{z_{\infty} w_{l}}\right\|_{\infty}<1
$$

The following lemma gives the necessary and sufficient condition for the existence of the $\mathrm{H}_{2}$ based SOF controller to meet the following performance criteria. 


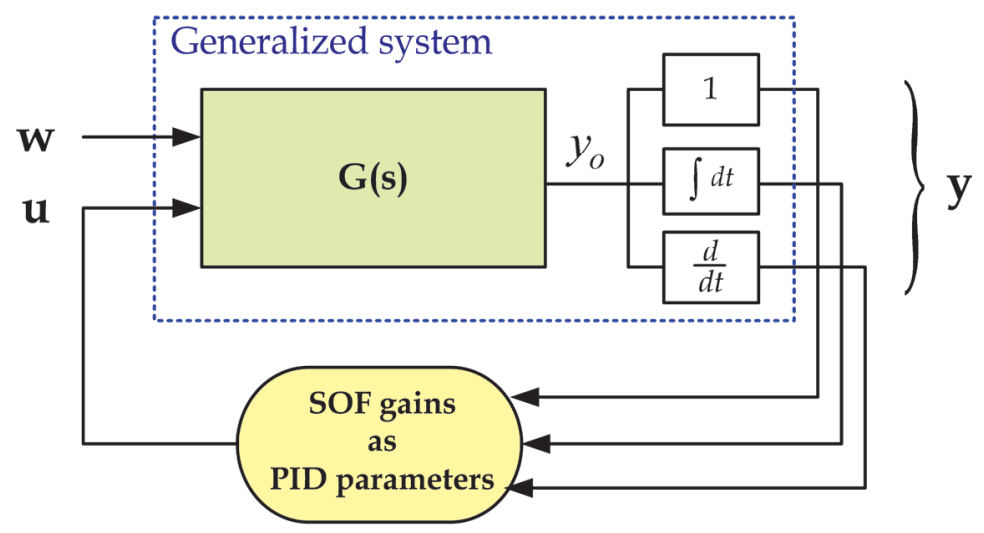

Fig. 1. PID as SOF control.

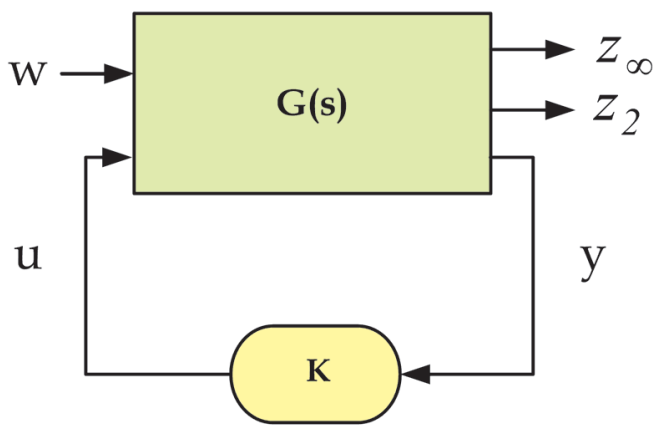

Fig. 2. Closed-loop system via mixed $\mathrm{H}_{2} / \mathrm{H}_{\infty}$ control.

$$
\left\|T_{z_{2} w_{2}}\right\|_{2}<\gamma_{2}
$$

where, $\gamma_{2}$ is the $\mathrm{H}_{2}$ optimal performance index, which demonstrates the minimum upper bound of $\mathrm{H}_{2}$ norm and specifies the disturbance attention level.

The $\mathrm{H}_{2}$ and $\mathrm{H}_{\infty}$ norms of a transfer function matrix $\mathrm{T}(\mathrm{s})$ with $\mathrm{m}$ lines and $\mathrm{n}$ columns, for a MIMO system are defined as:

$$
\begin{gathered}
\|T(s)\|_{2}=\sqrt{\sum_{j=1}^{n} \sum_{i=1}^{m}\left\|T_{i j}(s)\right\|_{2}^{2}} \\
\|T(s)\|_{\infty}=\underset{w}{\operatorname{Sup} \max \sigma[T(j w)]}
\end{gathered}
$$

where, $\sigma$ is represents the singular values of $T(j w)$.

Lemma 1, (Zheng et al., 2002):

For fixed $\left(A, B_{1}, B_{2}, C_{y}, K\right)$, there exists a positive definite matrix $X$ which solves inequality 


$$
\begin{aligned}
& \left(A+B_{2} K C_{y}\right) X+X\left(A+B_{2} K C_{y}\right)^{T}+B_{1} B_{1}^{T}<0 \\
& X>L_{C}
\end{aligned}
$$

to satisfy (9), if and only if the following inequality has a positive definite matrix solution,

$$
\begin{aligned}
& A X+X A^{T}-X C_{y}{ }^{T} C_{y} X \\
& +\left(B_{2} K+X C_{y}{ }^{T}\right)\left(B_{2} K+X C_{y}{ }^{T}\right)^{T}+B_{1} B_{1}^{T}<0
\end{aligned}
$$

where $L_{C}$ in (12) denotes the controllability gramian matrix of the pair $\left(A_{c}, B_{I c}\right)$ and can be related to the $\mathrm{H}_{2}$ norm presented in (9) as follows.

$$
\left\|T_{z_{2} w_{2}}\right\|_{2}^{2}=\operatorname{trace}\left(C_{2 c} L_{C} C_{2 c}{ }^{T}\right)
$$

It is notable that the condition that $A+B_{2} K C_{y}$ is Hurwitz is implied by inequality (12). Thus if

$$
\operatorname{trace}\left(C_{2 c} X C_{2 c}{ }^{T}\right)<\gamma_{2}{ }^{2}
$$

the requirement (9) is satisfied.

Lemma 2, (Cao et al., 1998)

The system (A, B, C) is stabilizable via static output feedback if and only if there exists $P>0$, $X>0$ and $K$ satisfying the following quadratic matrix inequality

$$
\left[\begin{array}{cc}
A^{T} X+X A-P B B^{T} X-X B B^{T} P+P B B^{T} P & \left(B^{T} X+K C\right)^{T} \\
B^{T} X+K C & -I
\end{array}\right]<0
$$

In the proposed control strategy, to design the PI/PID multiobjective controller, the obtained SOF control problem to be considered as a mixed $\mathrm{H}_{2} / \mathrm{H}_{\infty}$ SOF control problem. Then to solve the yielding nonconvex optimization problem, which cannot be directly achieved by using LMI techniques, an ILMI algorithm is developed.

The optimization problem given in (8) defines a robust performance synthesis problem where the $\mathrm{H}_{2}$ norm is chosen as a performance measure. Recently, several LMI-based methods are proposed to obtain the suboptimal solution for the $\mathrm{H}_{2}, \mathrm{H}_{\infty}$ and/or $\mathrm{H}_{2} / \mathrm{H}_{\infty} \mathrm{SOF}$ control problems. It is noteworthy that using lemma 1, it is difficult to achieve a solution for (13) by the general LMI, directly. Here, to get a simultaneous solution to meet (9) and $\mathrm{H}_{\infty}$ constraint, and to get a desired solution for the above optimization problem, an ILMI algorithm is introduced which is well-discussed in (Bevrani \& Hiyama, 2007). The developed algorithm formulates the $\mathrm{H}_{2} / \mathrm{H}_{\infty}$ SOF control through a general SOF stabilization. In the proposed strategy, based on the generalized static output stabilization feedback lemma (lemma 2), first the stability domain of gain vector (PID parameters) space, which guarantees the stability of the closed-loop system, is specified. In the second step, the subset of the stability domain in the PID parameter space in step one is specified so that minimizes the $\mathrm{H}_{2}$ performance indix. Finally and in the third step, the design problem is reduced to find a point in the previous subset domain, with the closest $\mathrm{H} 2$ performance index to the optimal one which meets the $\mathrm{H}_{\infty}$ constraint. In summary, the proposed algorithm searches a 
desired mixed $\mathrm{H}_{2} / \mathrm{H}_{\infty}$ SOF controller $K \in K_{\text {sof }}$ within a family of $\mathrm{H}_{2}$ stabilizing controllers $K_{\text {sof }}$, such that

$$
\left|\gamma_{2}^{*}-\gamma_{2}\right|<\varepsilon, \gamma_{\infty}=\left\|T_{z_{\infty} w_{l}}\right\|_{\infty}<1
$$

where $\varepsilon$ is a small real positive number, $\gamma_{2}^{*}$ is $\mathrm{H}_{2}$ performance corresponded to the $\mathrm{H}_{2} / \mathrm{H}_{\infty}$ SOF controller $K_{i}$ and $\gamma_{2}$ is the reference optimal $\mathrm{H}_{2}$ performance index provided by application of standard $\mathrm{H}_{2} / \mathrm{H}_{\infty}$ dynamic output feedback control. The key point is to formulate the $\mathrm{H}_{2} / \mathrm{H}_{\infty}$ problem via the generalized static output stabilization feedback lemma such that all eigenvalues of $(A+B K C)$ shift towards the left half-plane through the reduction of $a$, a real negative number, to close to feasibility of (8). Infact, the $a$ shows the pole region for the closed-loop system. The developed ILMI algorithm is summarized in Fig. 3 (Bevrani \& Hiyama, 2007; Bevrani, 2009). The application of above methodology in automatic generation control for a multi-area power system is given in section 4 .

\section{Multi-objective GA-based PID tuning}

\subsection{Intelligent methodologies}

The intelligent technology offers many benefits in the area of complex and nonlinear control problems, particularly when the system is operating over an uncertain operating range. Generally for the sake of control synthesis, nonlinear systems are approximated by reduced order dynamic models, possibly linear, that represent the simplified dominant systems' characteristics. However, these models are only valid within specific operating ranges, and a different model may be required in the case of changing operating conditions. On the other hand, classical and nonflexible PID designs may not represent desirable performance over a wide range of operating conditions. Therefore, more flexible and intelligent PID synthesis approaches are needed.

In recent years, following the advent of modern intelligent methods, such as artificial neural networks (ANNs), fuzzy logic, multi-agent systems, GAs, expert systems, simulated annealing, Tabu search, particle swarm optimization, Ant colony optimization, and hybrid intelligent techniques, some new potentials and powerful solutions for PID tuing have arisen.

In control configuration point of view, the most proposed intelligent based PID tuning mechanisms are used for tuning the parameters of existing fixed structure PID controller as conceptually shown in Fig. 4. In Fig. 4, it is assumed that the system is controllable and can be stabilized via a PID controller. Here, the applied intelligent technique performs an automatic tuner. The initial values for the parameters of the fixed-structure controller $\left(k_{P}\right.$, $k_{I}$ and $k_{D}$ gains in PID) must first be defined. The trial-error and the widely used ZieglerNichols tuning rules are usually employed to set initial gain values according to the openloop step response of the plant. The intelligent technique collects information about the system response and recommends adjustments to be made to the PID gains. This is an iterative procedure until the fastest possible critical damping for the controlled system is achieved. The main components of the intelligent tuner include a response recognition unit to monitor the controlled response and extract knowledge about the performance of the current PID gain setting, and an embedded unit to suggest suitable changes to be made to the PID gains. 


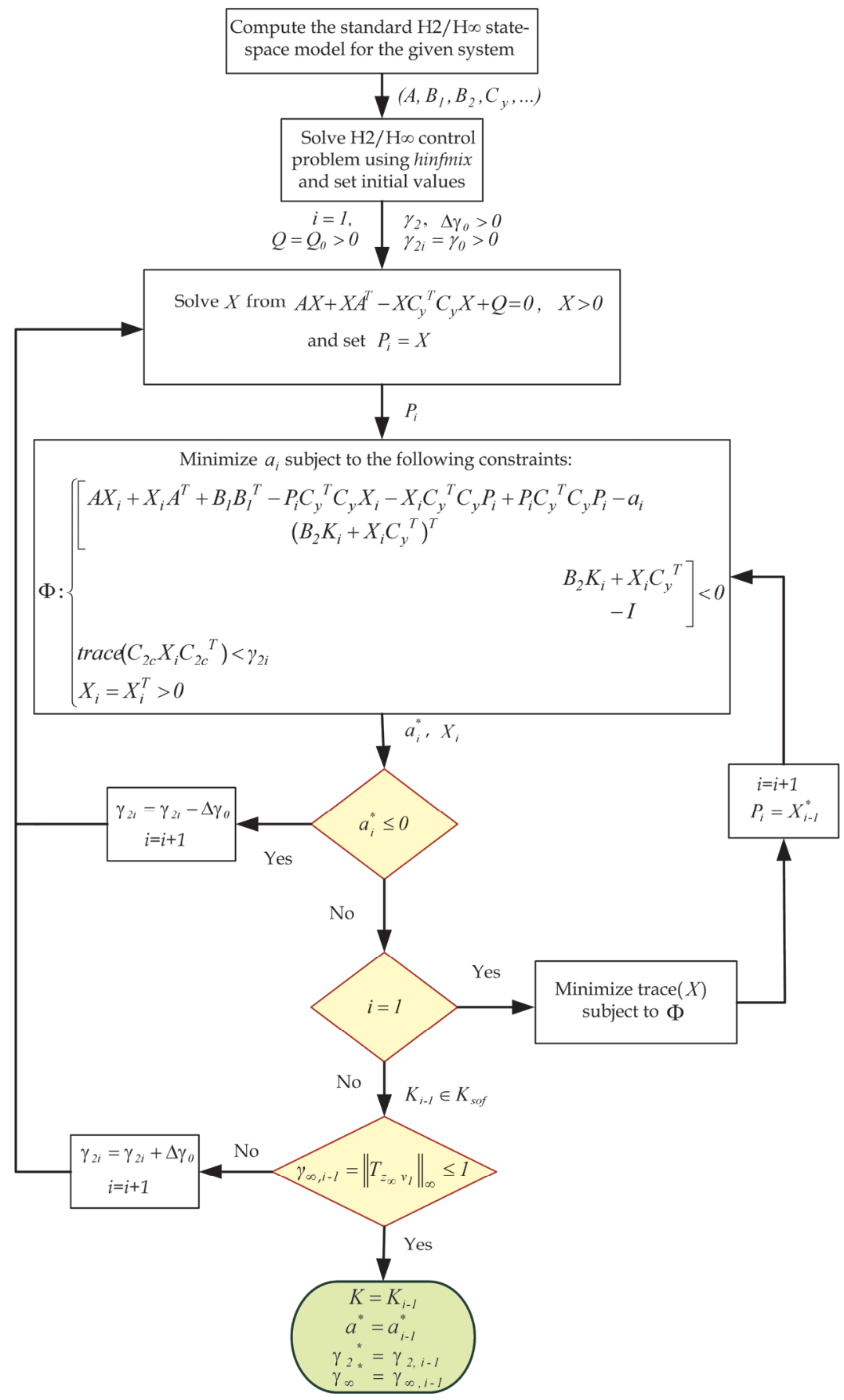

Fig. 3. Developed ILMI algorithm. 


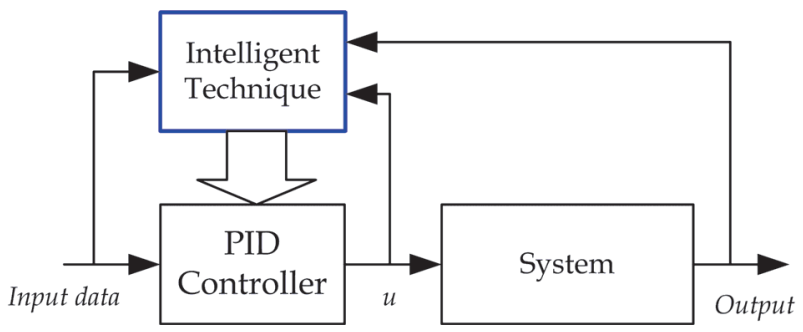

Fig. 4. Common configurations for intelligent-based PID designs.

\subsection{Genetic algorithm}

Genetic algorithm (GA) is a searching algorithm which uses the mechanism of natural selection and natural genetics; operates without knowledge of the task domain, and utilizes only the fitness of evaluated individuals. The GA as a general purpose optimization method has been widely used to solve many complex engineering optimization problems, over the years. In Fact, GA as a random search approach which imitates natural process of evolution is appropriate for finding global optimal solution inside a multidimensional searching space. From random initial population, GA starts a loop of evolution processes in order to improve the average fitness function of the whole population. GAs have been used to adjust parameters for different control schemes, e.g. integral, PI, PID, sliding mode control, or variable structure control (Bevrani \& Hiyama, 2007). The overall control framework for PID controllers is shown in Fig. 5.

Genetic algorithm (GA) is capable of being applied to a wide range of optimization problems that guarantees the survival of the fittest. Time consumption methods such as trial and error for finding the optimum solution cause to the interest on the meta-heuristic method such as GA. The GA becomes a very useful tool for tuning of parameters in PI/PID based control systems.

GA mechanism is inspired by the mechanism of natural selection where stronger individuals would likely be the winners in a competing environment. Normally in a GA, the parameters to be optimized are represented in a binary string. A simplified flowchart for GA is shown in Fig. 6. The cost function which determines the optimization problem represents the main link between the problem at hand (system) and GA, and also provides the fundamental source to provide the mechanism for evaluating of algorithm steps. To start the optimization, GA uses randomly produced initial solutions created by random number generator. This method is preferred when a priori information about the problem is not available. There are basically three genetic operators used to produce a new generation. These operators are selection, crossover, and mutation. The GA employs these operators to converge at the global optimum. After randomly generating the initial population (as random solutions), the GA uses the genetic operators to achieve a new set of solutions at each iteration. In the selection operation, each solution of the current population is evaluated by its fitness normally represented by the value of some objective function, and individuals with higher fitness value are selected (Bevrani \& Hiyama, 2011).

Different selection methods such as stochastic selection or ranking-based selection can be used. In selection procedure the individual chromosome are selected from the population for the later recombination/crossover. The fitness values are normalized by dividing each one by the sum of all fitness values named selection probability. The chromosomes with higher selection probability have a higher chance to be selected for later breeding. 
The crossover operator works on pairs of selected solutions with certain crossover rate. The crossover rate is defined as the probability of applying crossover to a pair of selected solutions (chromosomes). There are many ways to define the crossover operator. The most common way is called the one-point crossover. In this method, a point (e.g, for given two binary coded solutions of certain bit length) is determined randomly in two strings and corresponding bits are swapped to generate two new solutions.

Mutation is a random alteration with small probability of the binary value of a string position, and will prevent GA from being trapped in a local minimum. The coefficients assigned to the crossover and mutation specify number of the children. Information generated by fitness evaluation unit about the quality of different solutions is used by the selection operation in the GA. The algorithm is repeated until a predefined number of generations has been produced. Unlike the gradient-based optimization methods, GAs operate simultaneously on an entire population of potential solutions (chromosomes or individuals) instead of producing successive iterates of a single element, and the computation of the gradient of the cost functional is not necessary (Bevrani \& Hiyama, 2011).

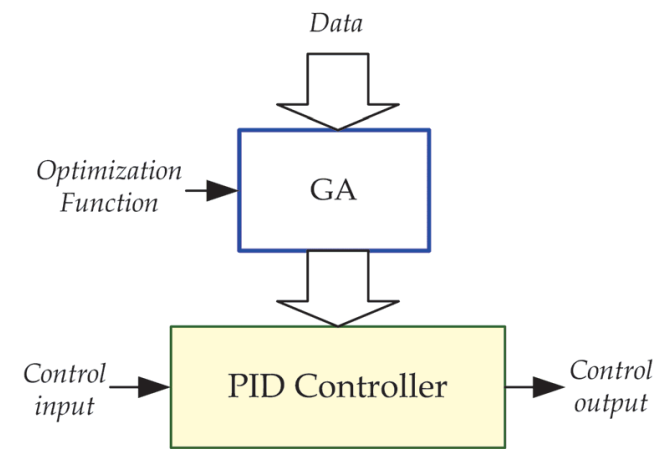

Fig. 5. GA-based PID tuning scheme.

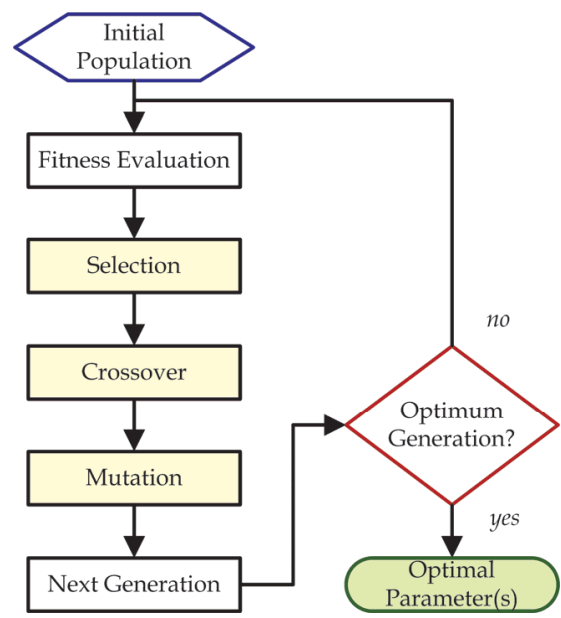

Fig. 6. A simplified GA flowchart. 
Several approaches are given for the analysis and proof of the convergence behavior of GAs. The proof of convergence is an important step towards a better theoretical understanding of GAs. Some proposed methodologies are based on building blocks idea and schema theorem (Thierens \& Goldberg, 1994; Holland, 1998; Sazuki, 1995).

\subsection{Multi-objective GA-based tuning mechanism}

The majority of PID control design problems are inherently multi-objective problems, in that there are several conflicting design objectives which need to be simultaneously achieved in the presence of determined constraints. If these synthesis objectives are analytically represented as a set of design objective functions subject to the existing constraints, the synthesis problem could be formulated as a multi-objective optimization problem.

In a multi-objective problem unlike a single optimization problem, the notation of optimality is not so straightforward and obvious. Practically in most cases, the objective functions are in conflict and show different behavior, so the reduction of one objective function leads to the increase in another. Therefore, in a multi-objective optimization problem, there may not exist one solution that is best with respect to all objectives. Usually, the goal is reduced to set compromising all objectives and determine a trade-off surface representing a set of nondominated solution points, known as Pareto-optimal solutions. A Pareto-optimal solution has the property that it is not possible to reduce any of the objective functions without increasing at least one of the other objective functions (Bevrani \& Hiyama, 2011).

Mathematically, a multi-objective optimization (in form of minimization) problem can be expressed as,

$$
\begin{aligned}
& \text { Minimize } y=f(x)=\left\{f_{1}(x), f_{2}(x), \ldots, f_{M}(x)\right\} \\
& \text { Subject to } g(x)=\left\{g_{1}(x), g_{2}(x), \ldots, g_{l}(x)\right\} \leq 0
\end{aligned}
$$

where $x=\left\{x_{1}, x_{2}, \ldots, x_{N}\right\} \in X$ is the vector of decision variables in the decision space $X$, $y=\left\{y_{1}, y_{2}, \ldots, y_{N}\right\} \in Y$ is the objective vector in the objective space. Practically, since there could be a number of Pareto-optimal solutions and the suitability of one solution may depends on system dynamics, environment, the designer's choice, etc., finding the center point of Pareto-optimal solutions set may be desired.

GA is well suited for solving of multi-optimization problems. In the most common method, the solution is simply achieved by developing a population of Pareto-optimal or near Pareto-optimal solutions which are nondominated. The $x^{\mathrm{i}}$ is said to be nondominated if there does not exist any $x \mathrm{j}$ in the population that dominates $x^{\mathrm{i}}$. Nondominated individuals are given the greatest fitness, and individuals that are dominated by many other individuals are given a small fitness. Using this mechanism, the population evolves towards a set of nondominated, near Pareto-optimal individuals (Fonseca \& Fleming, 1995). The multiobjective GA methodology is conducted to optimize the PID parameters. Here, the control objective is summarized to minimize the error signal in the control system. To achieve this goal and satisfy an optimal performance, the parameters of the PID controller can be selected through minimization of following objective function:

$$
O b j F n c=\int_{0}^{\mathrm{L}}|e(\tau)| d \tau \quad ; \quad e(t)=y(t)-y_{r}(t)
$$

where, ObjFnc is the objective function of control system, $L$ is equal to the simulation time duration (sec), $y_{r}(t)$ is the reference signal, and $|e(t)|$ is the absolute value of error signal at 
time $t$. Following using multi-objective GA optimization technique to tune the PID controller and find the optimum value of objective function (18), the fitness function (FitFunc) can be also defined as objective control function. Each GA individual is a double vector presenting PID parameters. Since, a PID controller has three gain parameters, the number of GA variables could be $N_{\text {var }}=3$. The population should be considered in a matrix with size of $m \times N_{\text {var }}$; where the $m$ represents individuals.

The basic line of the algorithm is derived from a GA, where only one replacement occurs per generation. The selection phase should be done, first. Initial solutions are randomly generated using a uniform random number of PID control parameters. The crossover and mutation operators are then applied. The crossover is applied on both selected individuals, generating two childes. The mutation is applied uniformly on the best individual. The best resulting individual is integrated into the population, replacing the worst ranked individual in the population. This process is conceptually shown in Fig. 7.

\section{Application to AGC design}

To illustrate the effectiveness of the introduced PID tuning strategies decribed in sections 2 and 3, the autumatic genertion control (AGC) synthesis for an interconnected three control areas power system, is considered as an example. AGC in a power system automaticaly minimizes the system frequency deviation and tie-line power fluctuation due to imballance between total generation and load, following a disturbance. AGC has a fundamental role in modern power system control/operation, and is well-disscussed in (Bevrani 2009, Bevrani \& Hiyama 2011). The power system configuration, data and parameters are given in (Rerkpreedapong et al., 2003). Each control area is approximated to a $9^{\text {th }}$ order linear system which includes three generating units.

\subsection{Mixed $\mathrm{H}_{2} / \mathrm{H}_{\infty}$ approach}

According to (5), the state-space model for each control area can be calculated as follows:

$$
\begin{aligned}
& \dot{x}_{i}=A_{i} x_{i}+B_{I_{i}} w_{i}+B_{2 i} u_{i} \\
& z_{\infty i}=C_{\infty i} x_{i}+D_{\infty 1_{i}} w_{i}+D_{\infty 2 i} u_{i} \quad i=1,2,3 \\
& z_{2 i}=C_{2 i} x_{i}+D_{21_{i}} w_{i}+D_{22_{i}} u_{i} \\
& y_{i}=C_{y i} x_{i}+D_{y I_{i}} w_{i}
\end{aligned}
$$

$y_{i}$ is the measured output (performed by area control error-ACE and its derivative and integral), $u_{i}$ is the control input and $w_{i}$ includes the perturbed and disturbance signals in the given control area.

The $\mathrm{H}_{2}$ controlled output signals in each control area includes $\Delta f_{i}, A C E_{i}$ and $\Delta P_{c i}$ which are frequency deviation, ACE (measured output) and governor load setpoint, respectively. The $\mathrm{H}_{2}$ performance is used to minimize the effects of disturbances on area frequency, ACE and penalize fast changes and large overshoot in the governor load set-point. The $\mathrm{H}_{\infty}$ performance is used to meet the robustness against specified uncertainties and reduction of its impact on the closed-loop system performance (Bevrani, 2009). First, a mixed $\mathrm{H}_{2} / \mathrm{H}_{\infty}$ dynamic controller is designed for each control area, using hinfmix function in the LMI control toolbox of MATLAB software. In this case, the resulted controller is dynamic type, whose order is the same as size of generalized plant model. Then, according to the tuning 


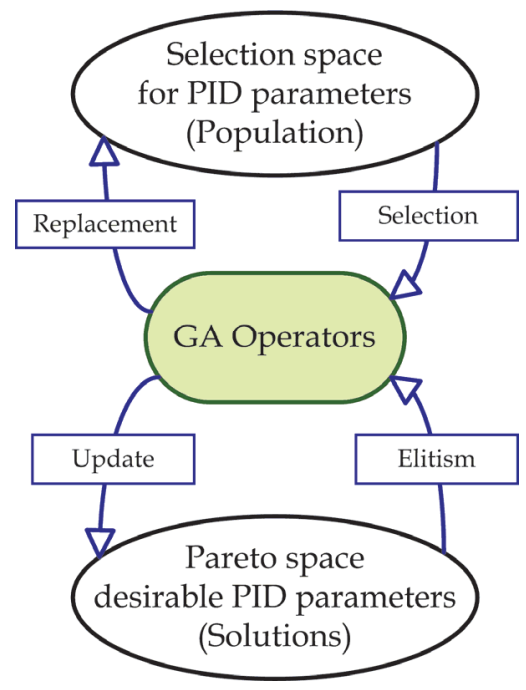

Fig. 7. Multi-objective GA for tuning of PID parameters.

methodology described in section 2, a set of three decentralized robust PID controllers are designed. Using developed ILMI algorithm, the controllers are obtained following several iterations. The proposed control parameters, the guaranteed optimal $\mathrm{H}_{2}$ and $\mathrm{H}_{\infty}$ indices $\left(\gamma_{2 i}\right.$ and $\left.\gamma_{\infty i}\right)$ for dynamic/PID controllers, and simulation results are shown in section 4.3.

It is noteworthy that here the design of dynamic controller is not a gole. However, the performance indeces of robust dynamic controller are used as valid (desirable) refrences to apply in the developed ILMI algorithm. It is shown that although the proposed ILMI approach gives a set of much simpler controllers (PID) than the dynamic $\mathrm{H}_{2} / \mathrm{H}_{\infty}$ design, however they holds robustness as well as dynamic $\mathrm{H}_{2} / \mathrm{H}_{\infty}$ controllers.

\subsection{GA approach}

The multi-objective GA-based tuning goal is summarized to minimize the area control error (ACE) signals in the interconnected control areas. Usally, the ACE signal is a linear combination of frequency deviation and tie-line power change (Bevrani, 2009). To achieve this goal, the objective function in a control is considered as

$$
\operatorname{ObjFnc}_{i}=\sum_{t=0}^{L}\left|A C E_{i, t}\right|
$$

where, $\left|A C E_{i, t}\right|$ is the absolute value of ACE signal for area $i$ at time $t$, and the fitness function is defined as follows,

$$
\operatorname{ObjFnc}(.)=\left[\operatorname{ObjFnc}_{1}, \mathrm{ObjFnc}_{2}, \ldots, \mathrm{ObjFnc}_{n}\right]
$$

Here, the number of GA variables is $N_{\text {var }}=3 n$, where $n$ is the number of control areas. 


\subsection{Simulation results}

The above described tuning approaches are applied to the 3-control area power system example. Fig. 8 shows the closed-loop response (ACE signals) for three areas, in the presence of simultaneous $0.1 \mathrm{pu}$ step load disturbances, and 20\% decrease in inertia constant and damping coefficient as uncertainties in all areas. Simulation results demonstrate that the GA-based tuning method is able to track the load fluctuations and meet robustness for a serious load disturbances as well as robust mixed $\mathrm{H}_{2} / \mathrm{H}_{\infty}$ tuning methodology. Interested readers can find more time domain simulations for various load disturbance scenarios in (Bevrani \& Hiyama, 2011).
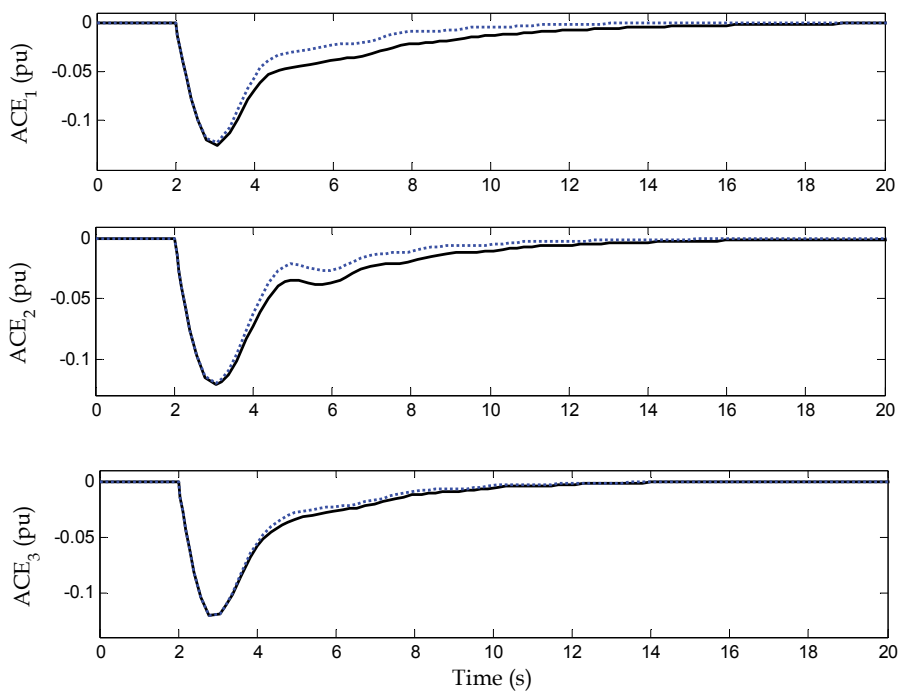

Fig. 8. Closed-loop system response; solid (GA), dotted (ILMI).

A new combination of these two tuning approaches is also introduced in (Bevrani \& Hiyama, 2011), which uses the GA to achieve the same robust performance indices $\left(\gamma_{2}^{*}, \gamma_{\infty}^{*}\right)$ as obtained via mixed $\mathrm{H}_{2} / \mathrm{H}_{\infty}$ control technique. In the proposed approach, the GA is employed as an optimization engine to produce the PID controllers with performance indices near to optimal ones.

\section{Fuzzy logic and PSO-based PID tuning}

\subsection{Overall framework}

Nowadays, fuzzy logic because of simplicity, robustness and reliability is used in almost all fields of science and technology, including solving a wide range of control and tuning problems. Unlike the traditional tuning methodologies, which are essentially based on the linearized mathematical models of the controlled systems, the fuzzy-based tuning technique tries to tune the controller parameters directly based on the measurements, long-term experiences and the knowledge of domain experts/operators. 
This section addresses a new intelligent methodology using a combination of fuzzy logic and particle swarm optimization (PSO) techniques to tune the parameters of PID controllers. The control parameters, $\mathrm{K}_{\mathrm{P}}, \mathrm{K}_{\mathrm{I}}$ and $\mathrm{K}_{\mathrm{D}}$, are automatically tuned using fuzzy rules, according to the on-line information. The PSO technique is used to find optimal values for membership functions parameters of the fuzzy logic scheme. The overall control framework is shown in Fig. 9.

\subsection{Tuning scheme}

As already mentioned, to improve the performance of PID controllers against changing of operating condition and system parameters, a fuzzy-based tuning mechanism can be able to adapt the PID parameters during the system operation and according to the on-line information. Such controllers are generally known as Two-level Controllers, or Gain Scheduling PID Controllers. In a two-level PID controller, usually the lower level controller (PID controller) performs fast direct control and higher level controller (fuzzy logic system as a supervisor) performs low speed supervision.

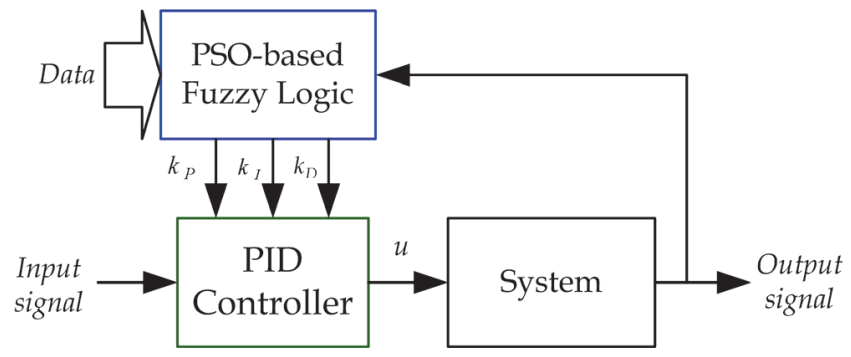

Fig. 9. Fuzzy logic for tuning of PID controller.

In the two-level Fuzzy-PID controller, direct control of the system (lower level) composed of a simple PID controller that generates the control signal $u(t)$ to apply to the plant as follows:

$$
u(t)=k_{P} e(t)+k_{I} \int e(t) d t+k_{D} \frac{d}{d t} e(t)
$$

where error signal $e(t)$ is used as input signal of the PID controller. Also, the fuzzy logic system acts as supervisor of PID controller performance and real-time tuning of its parameters according to system operating conditions.

Fig. 10, shows the structure of supervisory fuzzy system which is composed of four blocks. The fuzzification block represents the process of making crisp quantity into fuzzy. In fact, the fuzzifier converts the crisp input to a linguistic variable using the membership functions stored in the fuzzy knowledge base. Fuzzines in a fuzzy set is characterized by the membership functions. Using suitable membership functions, the ranges of input and output variables are assigned with linguistic variables. These variables transform the numerical values of the fuzzy unit input to the fuzzy quantities. These linguistic variables specify the quality of the control.

The concepts associated with a database are used to characterize fuzzy rules and a fuzzy data manipulation in fuzzy logic system. A lookup table is made based on discrete universes defines the output for all possible combinations of the input signals. A fuzzy system is 
characterized by a set of linguistic statements in the form of 'IF-THEN' rules. Fuzzy conditional statements make the rules or the rule set of the fuzzy system. Finally, the Inference engine uses the IF-THEN rules to convert the fuzzy input to the fuzzy output. On the other hand, defuzzifier converts the fuzzy output of the inference engine to crisp using membership functions analogous to the ones used by the fuzzifier. For defuzzification process, commonly center of sums, mean-max, weighted average and centroid methods are employed to defuzzify the fuzzy incremental control law (Bevrani \& Hiyama, 2011).

Generally, fuzzy logic design for a dynamical system involves the following four main steps:

Step 1: Understanding of the system dynamic behavior and characteristics. Define the states and input/output variables and their variation ranges,

Step 2: Identify appropriate fuzzy sets and membership functions. Create the degree of fuzzy membership function for each input/output variable and complete fuzzification,

Step 3: Define a suitable inference engine. Construct the fuzzy rule base, using the control rules that the system will operate under. Decide how the action will be executed by assigning strengths to the rules, and

Step 4: Determine defuzzification method. Combine the rules and defuzzify the output.

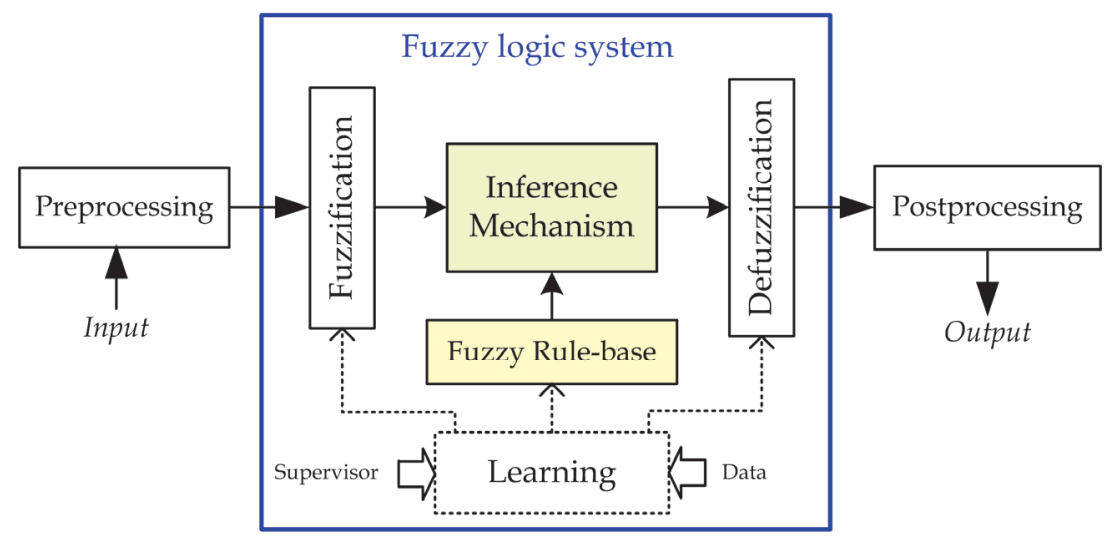

Fig. 10. A general scheme for fuzzy logic system.

Here, the PSO technique is used to perform the mentioned tuning mechanism. the PSO technique is used for tuning of fuzzy system's membership function parameters to improve the overall control performance (Bevrani \& Hiyama, 2011). The PSO is a population based stochastic optimization technique. In the PSO method, a swarm consists of a set of individuals, which each individual specified by position and velocity vectors $\left(x_{i}(t), v_{i}(t)\right)$ at each time or iteration. Each individual is named as a "particle" and the position of every particle represents a potential solution to the under study optimization problem. In an ndimensional solution space, each particle treated as a n-dimensional space vector and the position of the $i$-th particle is presented by $v_{i}=\left(x_{i 1}, x_{i 2}, \cdots, x_{i n}\right)$; then it flies to a new position by velocity represented by $v_{i}=\left(v_{i 1}, v_{i 2}, \cdots, v_{i n}\right)$. The best position for $i$-th particle represented by $p_{\text {best }, i}=\left(p_{\text {best }, i 1}, p_{\text {best }, i 2}, \cdots, p_{\text {best }, \text { in }}\right)$ is determined according to the best value obtained for the specified objective function. 
Furthermore, the best position found by all particles in the population (global best position), can be represented as $g_{\text {best }}=\left(g_{\text {best }, 1}, g_{\text {best }, 2}, \cdots, g_{\text {best }, n}\right)$. In each step, the best particle position, global position, and the corresponding objective function values should be saved. For the next iteration, the position $x_{i k}$ and velocity $v_{i k}$ corresponding to the $k$-th dimension of $i$-th particle can be updated using following equations:

$$
\begin{gathered}
v_{i k}(t+1)=w \cdot v_{i k}+c_{1} \cdot \operatorname{rand}_{1, i k}\left(p_{\text {best }, i k}(t)-x_{i k}(t)\right)+c_{2} \cdot \operatorname{rand}_{2, i k}\left(g_{\text {best }, k}(t)-x_{i k}(t)\right) \\
x_{i k}(t+1)=x_{i k}(t)+v_{i k}(t+1)
\end{gathered}
$$

where, $i=1,2, \ldots, n$ is the index of particles, $w$ is the inertia weight, $\operatorname{rand}_{1, i k}$ and $\operatorname{rand}_{2, i k}$ are random numbers in interval [ $\left[\begin{array}{ll}0 & 1\end{array}\right], c_{1}$ and $c_{2}$ are learning factors, and $t$ represents the iterations.

Usually, a standard PSO algorithm contains the following steps (Daneshmand, 2010):

Step 1: All particles are initialized via a random solution. In this step, each particle position and associated velocity are set by randomly generated vectors. Dimension of position should be generated within a specified interval, and the dimension of velocity vector should be also generated from a bounded domain using uniform distributions.

Step 2: Compute the objective function for the particles.

Step 3: Compare the value of the objective function for the present position of each particle with the value of objective function corresponding to the pre-specified best position, and replace the pre-specified best position by the present position, if it provides a better result.

Step 4: Compare the value of the objective function for the present best position with the value of the objective function corresponding to the global best position, and replace the present best position by the global best position, if it provides a better result.

Step 5: Update the position and velocity of each particle according to equations (24) and (25).

Step 6: Stop algorithm if the stop criterion is satisfied. Otherwise, go to step 2.

\subsection{Application example}

In order to investigate the efficiency of the proposed PID tuning strategy, a computer simulation has been conducted to design of PID-based AGC system for the standard 39-bus 10-generator test system, including three wind farms (Daneshmand, 2010). The obtained results are compared with the conventional fuzzy logic-based AGC system.

Here, $A C E$ is considered as input signal, and the provided control signal, $u(t)$ is used to change the set points of AGC participant generating units. To track a desirable AGC performance in the presence of high penetration wind power in a multi-area power system, a decentralized fuzzy logic based PID control design is proposed. Decreasing the frequency deviations due to fast changes in output power of wind turbines, and limiting tie-lines power interchanges in an acceptable range, following disturbances, are the main goals of this effort.

The Mamdani type inference system is applied, and symmetric 7-segments triangular membership functions are used for input and output variables. The membership functions are defined as zero $(\mathrm{ZO})$, large negative $(\mathrm{LN})$, medium negative $(\mathrm{MN})$, small negative $(\mathrm{SN})$, small positive (SP), medium positive (MP), and large positive (LP).

In order to reach fast response from the fuzzy system, all membership functions considered as triangular with the following mathematical definition: 


$$
\mu_{X}\left(x_{i}\right)=\max \left(0,1-\left|\frac{x-x_{i}}{c}\right|\right)
$$

where, $x$ and $c$ are the mean and spread of the fuzzy set $X$, respectively; and $x_{i}$ is a crisp variable. Fuzzy rule base is the basis of fuzzy logic operation to map input space to the output space. Here, a rule base including 49 fuzzy rules is considered (Table 1). The rule base works on vectors composed of $A C E$ and its gradient $d A C E$.

Since fuzzy rules are stated in terms of linguistic variables, crisp inputs should be also mapped to linguistic values using Fuzzification. The antecedent part of the rules composed of two parts, combined with fuzzy "AND" operators. The combination is done based on interpreting the "AND" operator by "Minimum" operation. Considering (26), the antecedent part of above statement may be defined as follows:

$$
\mu_{(A C E A N D d A C E)}(x, y)=\min \left(\mu_{A C E}(x), \mu_{d A C E}(y)\right)
$$

where $\mu_{(A C E A N D d A C E)}(x)$ is the membership value of antecedent part, and $\mu_{A C E}$ and $\mu_{d A C E}$ are the membership values of $A C E$ and $A A C E$, respectively.

Similarly, for computing the consequent of each rule, the membership function of "Mamdani Minimum" implication method can be represented by

$$
\mu_{M P}=\min \left(\mu_{(A C E A N D d A C E)}, \mu_{\Delta P_{c}}\right)
$$

where $\mu_{M P}$ denotes the membership function resulted by "Mamdani Minimum" implication, and $\mu_{(A C E A N D d A C E)}$ is the membership value of the related antecedent part.

In order to combine rules and make a decision based on the all given rules, the sum method is used. Finally, for converting output fuzzy set of the fuzzy system to a crisp value the centroid method is used for defuzzification (Daneshmand, 2010).

Each set of input membership functions can be specified by one parameter, $A C E_{\max }$ for $\mathrm{ACE}$ and $d A C E_{\max }$ for $\mathrm{dACE}$. Also, for control output variables, lower and upper limits should be specified for PID parameters of each controller. Therefore, totally eight parameters should be optimized for membership functions using PSO algorithm.

For the sake of PSO algorithm in the present AGC design, the number of particles, particles size, $v_{\min }, v_{\max }, c_{1}$, and $c_{2}$ are chosen as $10,6,-0.5,0.5,2.8$, and 1.3, respectively. Following use of PSO algorithm, the optimal values for membership function parameters can be easily obtained.

To investigate the performance of the proposed control strategy, a network with the same topology as the well-known IEEE 10 generators 39-bus system is considered as a test system. The system consists of 10 generators, 19 loads, 34 transmission lines, and 12 transformers. The power system is divided to three control areas. Single-line diagram, simulation parameters for the generators, loads, lines, and transformers of the test system are given in (Daneshmand, 2010). The desined PID controllers are responsible for producing appropriate control action signals according to the measured $A C E$ signals and their time derivatives ( $d A C E$ ).

For the present case study, the installed capacity includes 582.57 MW of conventional generation and 68.4 MW of average wind power generation (10\% penetration). To demonstrate the effectiveness of the proposed control design, some nonlinear simulations 
are performed in the SimPower environment of MATLAB software. In the simulations, the performance of the closed loop system using the designed fuzzy logic based controllers are compared with well-tuned conventional PID controllers.

As a serious test condition, three load disturbances (step increase in demand) are applied to control areas as simultaneous $6.66 \mathrm{pu}$ step load increase in each area at $5 \mathrm{sec}$. All unitized values in this paper are given based on the value of the largest generator nominal power, i.e. $150 \mathrm{MW}$. The simulation results are shown in Fig. 11. The ACE signals of the closed loop system for all areas are presented, following the applied load disturbances. These figures show the superior performance of the proposed fuzzy logic based AGC schemes to the conventional PID-based AGC designs in deriving area control error and frequency deviation close to zero. The PID parameters for conventional PID controllers in three control areas are listed in Table 2.

\begin{tabular}{cc|ccccccc}
\hline & \multicolumn{1}{c}{$d A C E$} \\
\cline { 3 - 8 } & & LN & MN & SN & ZO & SP & MP & LP \\
\hline \multirow{4}{*}{$A C E$} & LN & LP & LP & LP & MP & MP & SP & ZO \\
& NM & LP & MP & MP & MP & SP & ZO & SN \\
& SN & LP & MP & SP & SP & ZO & SN & MN \\
& ZO & MP & MP & SP & ZO & SN & MN & MN \\
& SP & MP & SP & ZO & SN & SN & MN & LN \\
& MP & SP & ZO & SN & MN & MN & MN & LN \\
& LP & ZO & SN & MN & MN & LN & LN & LN \\
\hline
\end{tabular}

Table 1. Fuzzy rule base.

\begin{tabular}{|c|c|c|c|}
\hline Area & $k_{P}$ & $k_{I}$ & $k_{D}$ \\
\hline$I$ & -0.852 & -1.724 & -0.001 \\
\hline$I I$ & -0.579 & -0.950 & -0.013 \\
\hline III & -0.971 & -1.900 & -0.007 \\
\hline
\end{tabular}

Table 2. Conventional PID parameters.

\section{Conclusion}

Most of real-world control problems refer to multi-objective control designs that several objectives such as stability, disturbance attenuation and reference tracking with considering practical constraints must be simultaneously followed usually by a simple PID controller. In such cases, multi-objective based tuning approaches are needed. In this direction, the present chapter addresses three powerfull robust/intelligent multi-objective methodologies to improve the performance of PID-based control systems.

The proposed approaches use mixed $\mathrm{H}_{2} / \mathrm{H}_{\infty}$, multi-objective genetic algorithm (GA), fuzzy logic, and particle swarm optimization (PSO) techniques as optimization tools for optimal tuning of PID parameters. Numerical examples on AGC design in multi-area power systems are given to illustrate the effectiveness of tuning methods. 

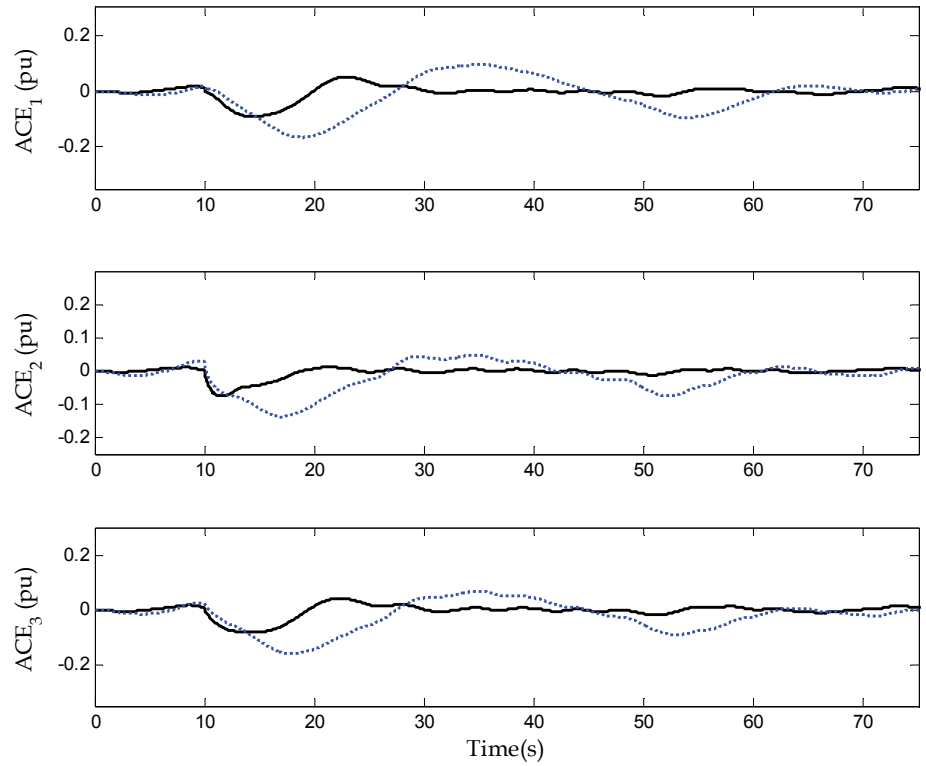

Fig. 11. ACE signals; proposed PID scheme (solid), conventional PID design (dotted).

\section{References}

Bevrani, H. \& Hiyama, T. (2007) Multiobjective PI/PID Control Design Using an Iterative Linear Matrix Inequalities Algorithm. International Journal of Control, Automation, and Systems, Vol.5, No.4, pp. 117-127.

Bevrani, H. (2009) Robust Power System Frequency Control. Springer, ISBN : 9780387848778, New York, USA.

Bevrani, H. \& Hiyama, T. (2011) Intelligent Automatic Generation Control. CRC Press (Taylor \& Francis Group), ISBN : 9781439849538, New York, USA.

Cao, Y.Y. ; Lam, J. ; Sun, Y. X. \& Mao, W. J. (1998) Static output feedback stabilization: an ILMI approach. Automatica, Vol. 34, No. 12, pp. 1641-1645.

Chen, B. S. ; Cheng, Y. M. \& Lee, C. H. (1998) A genetic approach to mixed $\mathrm{H}_{2} / \mathrm{H}_{\infty}$ optimal PID control. IEEE Control Systems, Vol. 15, No. 5, pp. 51-60.

Daneshmand, P. R. (2010) Power system frequency control in the presence of wind turbines, MSc. dissertation, Department of Electrical and Computer engineering, University of Kurdistan, Sanandaj, Iran, 2010.

Fonseca, C.M. \& Fleming, P.J. (1995) Multiobjective optimization and multiple constraint handling with evolutionary algorithms -part I: a unified formulation. IEEE Trans. Syst. Man \& Cybernetics, A, Vol.28, No.1, pp. 26-37.

Holland, J. H. (1998) Application in natural and artificial systems. The MIT Press, Combridge, Massachusetts, USA.

Rerkpreedapong, D ; Hasanovic, A. \& Feliachi, A. (2003) Robust load frequency control using genetic algorithms and linear matrix inequalities. IEEE Trans. On Power Systems, Vol. 18, No. 2, pp. 855-861. 
Rudolph, G (1997) Convergence properties of evolutionary algorithms. Kovac, Hamburg

Sazuki, J (1995) A markov chain analysis on simple genetic algorithms. IEEE Transactions on Systems, Man and Cybernetics, Vol.25, No.4, pp. 655-659.

Takahashi, R. H. C. ; Peres, P. L. D. \& Ferreira, P. A. V. (1997) Multiobjective $\mathrm{H}_{2} / \mathrm{H}_{\infty}$ guaranteed cost PID design. IEEE Control Systems, Vol. 17, No. 5, pp. 37-47.

Thierens, D. \& Goldberg, D. E. (1994) Convergence models of genetic algorithm selection schemes. Parallel problem solving from nature-PPSN III, pp.119-129.

Zheng, F. ; Wang, Q. G. \& Lee, H. T. (2002) On the design of multivariable PID controllers via LMI approach. Automatica, Vol. 38, pp. 517-526. 


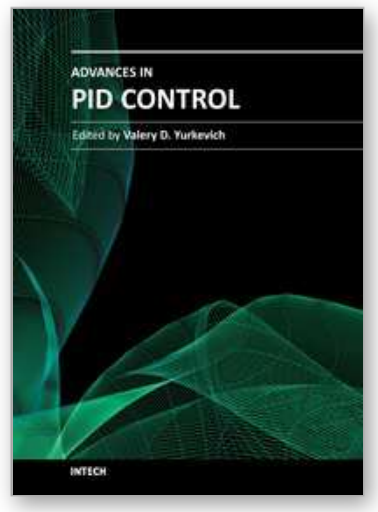

\author{
Advances in PID Control \\ Edited by Dr. Valery D. Yurkevich
}

ISBN 978-953-307-267-8

Hard cover, 274 pages

Publisher InTech

Published online 06, September, 2011

Published in print edition September, 2011

Since the foundation and up to the current state-of-the-art in control engineering, the problems of PID control steadily attract great attention of numerous researchers and remain inexhaustible source of new ideas for process of control system design and industrial applications. PID control effectiveness is usually caused by the nature of dynamical processes, conditioned that the majority of the industrial dynamical processes are well described by simple dynamic model of the first or second order. The efficacy of PID controllers vastly falls in case of complicated dynamics, nonlinearities, and varying parameters of the plant. This gives a pulse to further researches in the field of PID control. Consequently, the problems of advanced PID control system design methodologies, rules of adaptive PID control, self-tuning procedures, and particularly robustness and transient performance for nonlinear systems, still remain as the areas of the lively interests for many scientists and researchers at the present time. The recent research results presented in this book provide new ideas for improved performance of PID control applications.

\title{
How to reference
}

In order to correctly reference this scholarly work, feel free to copy and paste the following:

Hassan Bevrani and Hossein Bevrani (2011). PID Tuning: Robust and Intelligent Multi-Objective Approaches, Advances in PID Control, Dr. Valery D. Yurkevich (Ed.), ISBN: 978-953-307-267-8, InTech, Available from: http://www.intechopen.com/books/advances-in-pid-control/pid-tuning-robust-and-intelligent-multi-objectiveapproaches

\section{INTECH}

open science | open minds

\section{InTech Europe}

University Campus STeP Ri

Slavka Krautzeka 83/A

51000 Rijeka, Croatia

Phone: +385 (51) 770447

Fax: +385 (51) 686166

www.intechopen.com

\section{InTech China}

Unit 405, Office Block, Hotel Equatorial Shanghai

No.65, Yan An Road (West), Shanghai, 200040, China

中国上海市延安西路65号上海国际贵都大饭店办公楼 405 单元

Phone: +86-21-62489820

Fax: $+86-21-62489821$ 
(C) 2011 The Author(s). Licensee IntechOpen. This chapter is distributed under the terms of the Creative Commons Attribution-NonCommercialShareAlike-3.0 License, which permits use, distribution and reproduction for non-commercial purposes, provided the original is properly cited and derivative works building on this content are distributed under the same license. 